\title{
Elucidação estrutural de um complexo inédito de cobre(II) com 2-acetilpiridina etilcarbazato
}

\author{
G. S. Liarte ${ }^{\mathrm{a}}$, C. C. Gatto ${ }^{\mathrm{a}}$ \\ ${ }^{a}$ LASIC - Laboratório de Síntese Inorgânica e Cristalografia - Instituto de Química, \\ Universdade de Brasília, Brasília/DF, Brasil.
}

A bioinorgânica e a química de coordenação de metais de transição são áreas da química que estão em constante crescimento devido ao grande impacto que os seus resultados podem trazer para a sociedade. As pesquisas têm demonstrado que o estudo de complexos metálicos é importante para se encontrar alternativas para o combate a doenças e microorganismos. ${ }^{1,2}$

Os carbazatos, conforme estrutura genérica demonstrada na Figura 1, são bases Schiff que ainda não foram muito exploradas pela literatura científica. Entretanto, os carbazatos possuem estruturas muito semelhantes a outros ligantes que também são bases de Schiff e por isso, é possível presumir que eles possuirão grande capacidade farmacológica. ${ }^{3-6}$ Além disso, complexos derivados de ligantes carbazatos já foram reportados por possuírem grande capacidade antibacteriana e antifúngica. ${ }^{7}$

O estudo das semicarbazonas, por exemplo, pode ser útil ao avaliar estruturalmente os complexos derivados de carbazatos devido à presença de dois sítios de coordenação iguais nos dois tipos de ligante: o nitrogênio azometínico e o oxigênio carbonílico. Esta similaridade entre diferentes classes de ligante pode ajudar na avaliação de comprimentos e ângulos de ligação de complexos ao determinar geometrias ou na avaliação de dados espectroscópicos, por exemplo.

Muitos metais desempenham funções importantes nos seres vivos devido à possibilidade que apresentam de interagir com moléculas biológicas, como as proteínas e o DNA. Íons de cobre, por exemplo, fazem parte da estrutura da hemocianina, proteína responsável pelo fornecimento de oxigênio aos tecidos de artrópodes. Tudo isto associado ao fato de os carbazatos apresentarem variados modos de coordenação, faz com que o estudo dos complexos metálicos com ligantes carbazatos seja muito importante. ${ }^{8-10}$

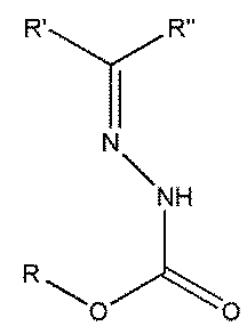

Figura 1. Estrutura genérica de carbazatos (sendo $R=$ grupos alquilas ou arilas e $R$ ' e $R$ ' $=H$, grupos alquilas ou arilas)

O presente trabalho apresenta o estudo da estrutura cristalina e molecular de um novo complexo de cobre(II), [Cu(apec) $\left.\mathrm{Br}_{2}\right] \mathrm{H}_{2} \mathrm{O}$, que foi obtido por meio de uma reação de complexação entre o ligante 2-acetilpiridina etilcarbazato (apec) e o sal brometo de cobre. Os dados principais da coleta e refinamento obtidos através da análise por difração de raios $\mathrm{X}$ do complexo $\left[\mathrm{Cu}(\mathrm{apec}) \mathrm{Br}_{2}\right] \mathrm{H}_{2} \mathrm{O}$ encontram-se na Tabela 1.

A estrutura cristalina do complexo $\left[\mathrm{Cu}(\mathrm{apec}) \mathrm{Br}_{2}\right] \mathrm{H}_{2} \mathrm{O}$ é formada por uma molécula do ligante apec coordenado de forma tridentada ao átomo de cobre por meio do nitrogênio piridínico, do nitrogênio azometínico e do oxigênio carbonílico. No poliedro de coordenação deste complexo ainda se encontram dois íons brometos coordenados ao centro metálico de cobre, fazendo com que este poliedro assuma uma geometria piramidal de base quadrada 
distorcida, além da presença de uma molécula de água de cristalização. A representação da estrutura deste complexo pode ser observada na Figura 2.

A geometria do poliedro de coordenação pode ser avaliada também segundo o parâmetro de Addison. ${ }^{11}$ Como o valor deste parâmetro está mais próximo de zero, é possível sugerir que a geometria do complexo é piramidal de base quadrada. É possível observar também através dos ângulos de ligação, da base da pirâmide formada, que variam entre $78,92(16)^{\circ} \mathrm{e}$ $104,71(4)^{\circ}$ para os átomos $\mathrm{N}(1)-\mathrm{Cu}(1)-\mathrm{N}(2), \mathrm{N}(2)-\mathrm{Cu}(1)-\mathrm{O}(1)$ e $\mathrm{Br}(2)-\mathrm{Cu}(1)-\mathrm{Br}(1)$, a distorção do poliedro de coordenação para o átomo de cobre(II). A Tabela 2 mostra alguns dos comprimentos e ângulos de ligação mais importantes para o complexo $\left[\mathrm{Cu}(\mathrm{apec}) \mathrm{Br}_{2}\right] \mathrm{H}_{2} \mathrm{O}$.

Tabela 1. Dados da coleta e refinamento por difração de raios $X$ de monocristais para o complexo [ $\mathrm{Cu}($ apec $\left.) \mathrm{Br}_{2}\right] \mathrm{H}_{2} \mathrm{O}$

\begin{tabular}{ll}
\hline Fórmula Molecular & $\mathrm{C}_{10} \mathrm{H}_{15} \mathrm{Br}_{2} \mathrm{CuN}_{3} \mathrm{O}_{3}$ \\
Massa Molar $\left(\mathrm{g} \mathrm{mol}^{-1}\right)$ & 448,61 \\
Sistema Cristalino & Triclínico \\
Grupo Espacial & $\mathrm{P}-1$ \\
Parâmetros de cela & \\
a $(\AA)$ & $7,940(4)$ \\
$\mathrm{b}(\AA)$ & $8,590(4)$ \\
c $(\AA)$ & $12,409(6)$ \\
$\alpha=82,67(3)^{\circ}$ & $82,67(3)^{\circ}$ \\
$\beta=87,04(3)^{\circ}$ & $87,04(3)^{\circ}$ \\
$\gamma=63,63(3)^{\circ}$ & $63,63(3)^{\circ}$ \\
Volume $\left(\AA^{3}\right)$ & $752,0(6)$ \\
$\mathrm{Z}$ & 2 \\
$\mathrm{R} 1 /$ wR2 & $0,0378 / 0,0907$ \\
\hline
\end{tabular}

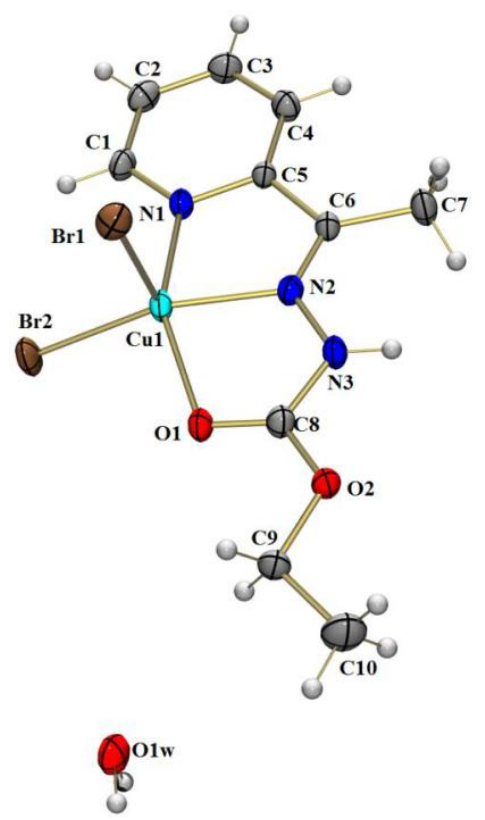

Figura 2. Diagrama ORTEP do complexo [Cu(apec) $\left.\mathrm{Br}_{2}\right] \mathrm{H}_{2} \mathrm{O}$. Os elipsoides térmicos se apresentam em um nível de probabilidade de $30 \%$.

No complexo, a forma ceto do ligante livre é mantida com a coordenação ao centro metálico e isto pode ser observado analisando os comprimentos da ligação entre $\mathrm{C}(8)-\mathrm{O}(1)$ no ligante livre e no complexo[Cu(apec) $\left.\mathrm{Br}_{2}\right] \mathrm{H}_{2} \mathrm{O}$, que são de 1,204(2) $\AA$ e 1,230(6) $\AA$, respectivamente. Essa pequena variação deste comprimento de ligação após a complexação indica que o ligante permaneceu na forma ceto e a sua variação pode ser explicada pela 
coordenação obtida, além de ter sido comprovada também por outras técnicas espectroscópicas de análise.

A estrutura cristalina do complexo $\left[\mathrm{Cu}(\operatorname{apec}) \mathrm{Br}_{2}\right] \mathrm{H}_{2} \mathrm{O}$ apresenta ainda duas ligações de hidrogênio intermoleculares entre os átomos $\mathrm{N}(3) \mathrm{H}(3 \mathrm{~A}) \cdots \mathrm{O}(1 \mathrm{~W}) \mathrm{i}$ e $\mathrm{O}(1 \mathrm{~W}) \mathrm{H}(1 \mathrm{WA}) \cdots \mathrm{Br}(1) \mathrm{ii}$, com distâncias $\mathrm{N}(3) \cdots \mathrm{O}(1 \mathrm{~W})$ de 2,753(6) $\AA$ e $\mathrm{O}(1 \mathrm{~W}) \cdots \mathrm{Br}(1)$ de 3,377(4) $\AA$, que são formadas através dos operadores de simetria (i): $\mathrm{x}, \mathrm{y}-1, \mathrm{z}$ e (ii): $-\mathrm{x}+1,-\mathrm{y}+1,-\mathrm{z}$, respectivamente. As ligações de hidrogênio formadas neste complexo estabilizam a estrutura cristalina como um todo e facilitam a obtenção de cristais adequados para análise de difração de raios $\mathrm{X}$ de monocristal.

Tabela 2. Principais comprimentos de ligação $(\AA)$ e ângulos $\left({ }^{\circ}\right)$ relativos ao poliedro de coordenação do complexo [Cu(apec) $\mathrm{Br}_{2} \cdot \mathrm{H}_{2} \mathrm{O}$ ]

Comprimentos de ligação $(\AA)$

$\mathrm{Cu}(1)-\mathrm{Br}(1)$

$\mathrm{Cu}(1)-\mathrm{Br}(2)$

$\mathrm{Cu}(1)-\mathrm{N}(2)$

$\mathrm{Cu}(1)-\mathrm{N}(1)$

$\mathrm{Cu}(1)-\mathrm{O}(1)$
$2,5825(14)$

$2,3535(13)$

$1,973(4)$

$2,020(4)$

$2,099(3)$

Ângulos de ligação $\left(^{\circ}\right)$

$\begin{array}{ll}\mathrm{N}(1)-\mathrm{Cu}(1)-\mathrm{N}(2) & 78,92(16) \\ \mathrm{N}(2)-\mathrm{Cu}(1)-\mathrm{O}(1) & 77,42(15) \\ \mathrm{O}(1)-\mathrm{Cu}(1)-\mathrm{Br}(2) & 97,91(10) \\ \mathrm{Br}(2)-\mathrm{Cu}(1)-\mathrm{Br}(1) & 104,71(4) \\ \mathrm{Br}(2)-\mathrm{Cu}(1)-\mathrm{N}(1) & 99,38(11)\end{array}$

O complexo $\left[\mathrm{Cu}(\mathrm{apec}) \mathrm{Br}_{2}\right] \mathrm{H}_{2} \mathrm{O}$ cristaliza no sistema cristalino triclínico e grupo espacial . A cela unitária é formada por duas unidades assimétricas do complexo e o único elemento de simetria presente nesta cela é o centro de inversão, como pode ser observado na representação da cela unitária da Figura 3.

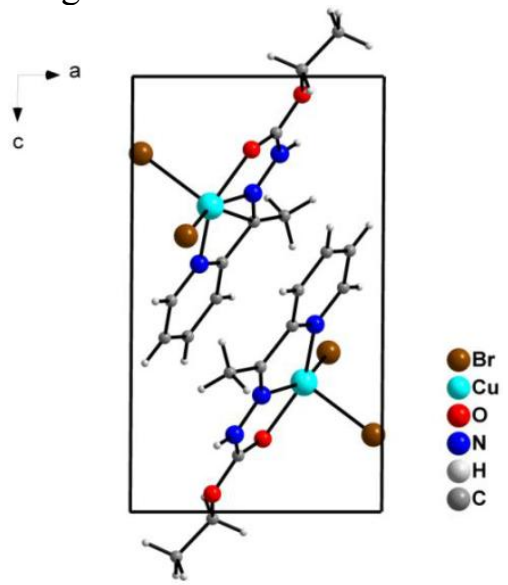

Figura 3. Representação da cela unitária do complexo $\left[\mathrm{Cu}(\right.$ apec $\left.) \mathrm{Br}_{2}\right] \mathrm{H}_{2} \mathrm{O}$.

Além da estrutura cristalina obtida por difração de raios X, também foram utilizadas outras técnicas de análise para caracterizar o complexo obtido. Foram feitas, então, análises de espectroscopia vibracional na região do infravermelho, espectroscopia de absorção da região do ultravioleta-visível, análise elementar e ponto de fusão.

Os resultados obtidos se mostraram muito interessantes devido à formação de um complexo inédito com um ligante que não apresenta estudos na literatura. Além disso, este estudo contribui para o desenvolvimento da química de coordenação e química bioinorgânica, pois as análises biológicas, contra bactérias e fungos, se encontram em desenvolvimento.

\section{Referências:}

[1] Montanari, C.; Quim. Nova, 2000, 23, 134.

[2] Selvamurugan, S.; Ramachandran, R.; Vijayan, P.; Manikandan, R.; Prakash, G.; Viswanathamurthi, P.; Velmurugan, K.; Nandhakumar, R.; Endo, A.; Polyhedron, 2016, 107, 57-67.

[3]- Zangrando, E.;Begum, M.; Sheikh, M.; Miyatake, R.; Hossain, M.; Alam, M.; Hasnat, M.; Halim, M.; Ahmed, S.; Rahman, M.; Ghost, A.; Arab J Chem, 2017, 10, 172. 
[4]Xu, J.;Zhou, T.; Xu, Z.; Gu, X.; Wu, W.; Chen, H.; Wang, Y.; Jia, L.; Zhu, T.; Chen, R.; J Mol Struct, 2017, $1128,448$.

[5]Venkatachalam, T.; Bernhardt, P.; Noble, C.; Fletcher, N.; Pierens, G.; Thurecht, K.; Reutens, D.; $J$ Inorg Biochem, 2016, 162, 295.

[6] Shaabani, B.; Khandar, A.; Mahmoudi, F.; Maestro, M.; Balula, S.; Silva, L.; Polyhedron, 2013, 57,118

[7] Milenkoviv, M.; Cantoni, G.; Bacchi, A.; Spasojevic, V.; Milenkovic ,M.; Sladic, D.; Krstic, N.; Andelkovic, K.; Polyhedron, 2014, 80, 47.

[8] Holde, K.; Miller, K.; Decker, H.; J Biol Chem, 2001, 276, 15563.

[9] Maqbool, A.; Herve, M.; Meguin-LecreulX, D.; Wilkinson, A.; Thomas, G.; Biochem J, 2012, 448, 329.

[10] Lv, J.; Jiang, Y.; Yu, Q.; Lu, S.; J BiollnorgChem, 2011, 16, 125

[11] Addison, A.; Rao, T.; Reedjick, J.; Rijn, J.; Verschoor, G.; J. Chem. Soc. Dalton Trans.; 1984, 7, 1349.

Agradecimentos: FAPDF; CAPES; CNPq; IQ-UnB. 